Proceedings

\title{
Images and Models of Thought ${ }^{+}$
}

\author{
Andrea Casale \\ School of Architecture, Department of History, Representation, and Restoration of Architecture, Sapienza \\ University of Rome, 00186 Roma, Italy; andrea.casale@uniroma1.it \\ + Presented at the International and interdisciplinary conference IMMAGINI? Image and Imagination \\ between representation, communication, education and psychology, Brixen, Italy, 27-28 November 2017.
}

Published: 17 November 2017

\begin{abstract}
One really extraordinary ability of the mind is its capacity to match objects and form plausible hypotheses from just a few elements that we see through our eyes. We recognize a feather even if it is mostly covered by a book sitting on top of it. Even if we cannot see the whole shape, we recognize it as pertaining to a category, a set of objects called "feathers". If by imagination we mean the ability to represent things for ourselves that are not present in the act of sensing, we should realize that the hypothesis of the feather is an imaginative construction of the mind, a mental representation, a model referred to by the sensory input.
\end{abstract}

Keywords: image; model; recognition; configuration; project

\section{Introduction}

Recognition is an operation in which bottom-up processes, which produce the mental model by acquiring information, coexist simultaneously and actively with top-down processes, in which the mental model looks for confirmation in sensory information. But if recognition is the result of the convergence of external information and imaginative mnemonic models, it means that models of reference resulting from abstraction should already exist in our mind. In 1896, Henri Bergson demonstrated the presence of a vicious cycle when he wrote, "In order to generalize, one must first abstract, but to abstract usefully, one should already know how to generalize" [1].

It is therefore inevitable that the brain should be capable of identifying recurring external events. Samir Zeki distinguishes between hereditary and acquired concepts [2] (pp. 17-45). The former result from the organization of abstractions deduced from arriving signals; the latter entails identification of concepts/categories to which the recognition of an object can be connected.

Hereditary concepts are determined by the solicitation of particular areas of the brain that are sensitive to some specific qualities of the stimulus. Some groups of neurons are activated by verticality and are indifferent to whether the verticality refers to a house, a pencil, a man, or a tree; they are sensitive only to this quality. Just as there is an area sensitive to verticality, there is also an area sensitive to horizontality, a right and a left obliquity, the colours red, blue, etc. The simultaneous activation of multiple areas determines more complex concepts such as a straight line, a curve, parallel or orthogonal lines, and shape, but also the movement and relative position of two elements. These are hereditary concepts because they are intimately connected to the evolution of the species and therefore do not change during the unique personal experience. According to the recognitionby-components (RBC) theory, visual input in our brains corresponds to a structural representation determined by combinations and relationships of simpler 2D and 3D shapes called geons (rectangle, circle, cone, cylinder, parallelepiped, etc.). Irving Biederman identifies 36 geons, simple forms that, when combined according to position, proportion, and orientation, allow the structure of any object to be created [3] (pp. 115-147). 
The recognition of groups of geons recurring in many objects determines them as pertaining to a family, a category. We therefore recognize the category "chair", "table", "pencil", etc. These concepts can change over time since each chair, table, or pencil is different, but they can anyway be included in an overall group. It is clear that memory participates in the construction of the concept/categories acquired because the operation is made precisely by comparing what is detected perceptually and what resides in the memory. The interacting bottom-up and top-down relationship is triggered, causing us to recognize the object. This implies that the more we experience, the more the categories of elements expand, and the more we can define further categories.

As well, naming a category leads to a greater capacity to categorize. A botanist is capable of distinguishing many more plants than a person who has not studied botany, and this obviously does not depend on a greater visual capacity. From the form of the plant the botanist first manages to distinguish the family, then the genus, species, subspecies, race, variety, etc., until arriving at a specific name. In some cases, this knowledge does not derive from direct experience but from a culture assimilated through other systems such as books, videos, or photographs.

The recognition process therefore implies that sensory experience constantly informs us and that the information itself becomes a heritage and active growth in distinguishing and judging what is experienced. But this explanation is only partially satisfactory. How is it that a baby of just a few months is capable of recognizing the being encountered on the street as like the pet dog at home when it is completely different with respect to size, colour, and coat length?

Susanne K. Langer writes: “The abstraction of form ... is probably not made by comparison of several examples ... nor by repeated impressions ... but is derived from some single instance under proper conditions of imaginative readiness; whereupon the visual form, once abstracted, is ... used interpretively wherever it will serve and as long as it will serve" [4] (pp. 379-392).

The concepts/categories we acquire hold substantial importance in how we refer to the world. They are synthetic mental models appropriate for recognition and are managed differently by the individual according to the circumstances; the complexity of the model varies as a function of the contingent needs. If we move through our house to reach the room where we left a book we want to read, we move within a model that is simplified enough that we can also use our minds for other different thoughts. If, however, we do not find the book there, we need to think about where we left it, so we construct richer models in our minds, enriching them in part with new perceptual information, adding more details to previous mnemonic models. The scope of comparing these models is to solve the problem, the missing determination of what was anticipated.

It follows that the mental model that we experience is fundamentally a model we already possess. New experiences are extremely rare in our world of perceptions and can therefore always be identified as an offshoot of a known model. Even if we encounter new environments, they obey forms that, even if not in the specific quantities and qualities, already fundamentally pertain to configurations that are basically familiar for their shape and culture.

But if imagination is necessary to build the mental model of the world that surrounds us, imagination is also the tool with which we presuppose different realities and it is always through the imagination that we build relationships with others.

\section{From the Mental Model to the Image}

The image has often been attributed to a particular affinity with perception in that both are twodimensional representations and the result of a geometrical/projection condition: the first on a surface, the second on the retina. They also have a mimetic capacity, i.e., the prerogative to resemble something pertaining to the sensible world. There is thus a connection between the object and its depiction, which does not consider a higher mental operation, but the evidence of conditions that would make both the object and its representation converge in a recognizable concept; this recognizability is found both objectively and immediately. Pliny the Elder recounts a debate between the greatest artists of Ancient Greece: Zausi and Parrasio. The first paints a bunch of grapes with such realism that the birds are confused and come to peck at it. The second paints a cloth that covers the painting and induces Zausi to ask what has been moved. Aside from who won the challenge 
(according to the report, it was Parrasio because he managed to confuse Zausi), it is clear that Pliny's tale refers to an opinion that the image should be absolutely mimetic, imitative, and realistic. But within the class "images" we can also include diagrams, ideograms, the plans and perspectives of a building, children's drawings, abstract paintings, etc., i.e., images that are rather far from mimetic realism.

So that there is a resemblance, it is necessary to realize the reflexive property in which A resembles $B$ since $B$ resembles $A$. The object is a three-dimensional entity that lives in a space we perceive through two mobile eyes situated in a rotating head on a moving body. This means that the mental model drawn from the world we live in is dynamic, mobile, and changeable. The image can resemble the object, but the object does not resemble the image. Nelson Goodman states, ".... picture, to represent an object, must be a symbol for it, stand for it, refer to it; and that no degree of resemblance is sufficient to establish the requisite relationship of reference" [5] (pp. 11-13). It is therefore clear that the aim of building an image in order to trick the observer can never be fulfilled. Even if one is faced with objective photographic evidence of a fact, the observer can only construct a more or less strong empathetic relationship with the author since a specific descriptive desire is rediscovered in the work that inevitably ties it to the author of the image.

Studies on mirror neurons, advanced by a group of scholars in Parma under the guidance of Giacomo Rizzolatti, have demonstrated that users partially relive the emotional event that produced the work. They experience a state of cerebral empathy with the work and its artist beyond space and time. The more the author intends to transmit an aesthetic representation through the work, the more users identify that desire in it and will experience the absolutely particular tie between themselves and the artist through the physicality of the work.

Even stronger is the empathetic relationship between the work and users when they are familiar with the techniques and means of expression. For a photographer looking at a photograph, a painter admiring a painting, an architect observing a plan, areas of the brain are activated by mirror neurons analogous to those activated by the artist, architect, etc., in order to mentally make the gestures that produced the sign.

However, as I propose below, if we refer to the image as the product of thought like a crucible in which the thought is formed, realism and imitation are not absolutely necessary; at most they can hold meaning that is instrumental in conveying concepts. This is precisely what artists have always done, where the image, even if figurative, becomes the way to determine meanings.

It is therefore appropriate to make a distinction between accidental and intentional images. Accidental images are the result of a particular physical/geometric condition: the reflection on a surface, the shadow produced by a light source, but also a cloud in the sky or a stain on the wall on which the mind, in a particular imaginative state, lingers in recognition. Intentional images are the result of an individual's action with the scope of building a particular relationship with other individuals: designs, diagrams, paintings, photographs, etc.

The intentional image is often associated with language, both of which are means designed to communicate a thought. Cognitivism is traditionally based on the hypothesis that the brain is a processor of information whose purpose is to establish the solution to a problem. It is a biocalculator of enormous processing capacity equipped with input structures - the sensory organs - and output such as language (verbal, iconic, musical, etc.). Cognitivism therefore presumes that if the contribution of input data is inevitable, the thought is the result of a primarily internal processing that is sometimes expressed externally through output systems. This hypothesis attributes a strong degree of autonomy to the thought, with everything occurring within our minds. Formed absolutely, the thought uses the communicational tool of language to break the mind's isolation.

In an interview at the Fondazione Collegio San Carlo di Modena in on 19 February 2016 entitled "Contro le immagini della mente. Linguaggio e pensiero tra natura e cultura" [6], Felice Cimatti proposed an alternative hypothesis called Fundamental anthropologic situation. "At the beginning, there is a unitary relational field which includes both the infant and the adult, but also the adult's thoughts about the child... The relational field thinks for the individual mind, in the sense that it provides schemes of thought, words, common places, images, and mechanisms that build the first 
thought apparatus of each human being". What follows is an absolutely inseparable relationship between thought and language. Cimatti reminds us that for Ludwig Wittgenstein, language is "the life form of human animals". Therefore language plays an efficient, productive role in formulating thought.

Many times throughout his A Treatise on Painting, Leonardo da Vinci compares painting to poetry. In the first book, under the title "The difference between painting and poetry" he confirms that "painting is mute and poetry is blind painting", thereby describing the usual ancient relationship between image and language. But after what was affirmed by Ludwig Wittgenstein, we should realize that the image is a form of life for human animals and that it is therefore indivisible from the relationship between thought and image.

This means that we think through the image and that the thought is configured due to the image. The image, equal to language, is fully and definitively expressed after a series of continuous, exchangeable interactions between the complete image and its creator.

Some examples may help to illustrate this communicational relationship between the artist and the image, presenting some problems that are solved precisely due to this exchangeable, dynamic relationship.

A first example is the demonstration of the Pythagorean theorem proposed by Euclid.

Figure 1A shows the problem. We would like to demonstrate that the sum of the areas of the green and purple squares, which are built based on the sides of the yellow right triangle, is equal to the area of the blue square built based on the hypotenuse of the same triangle.

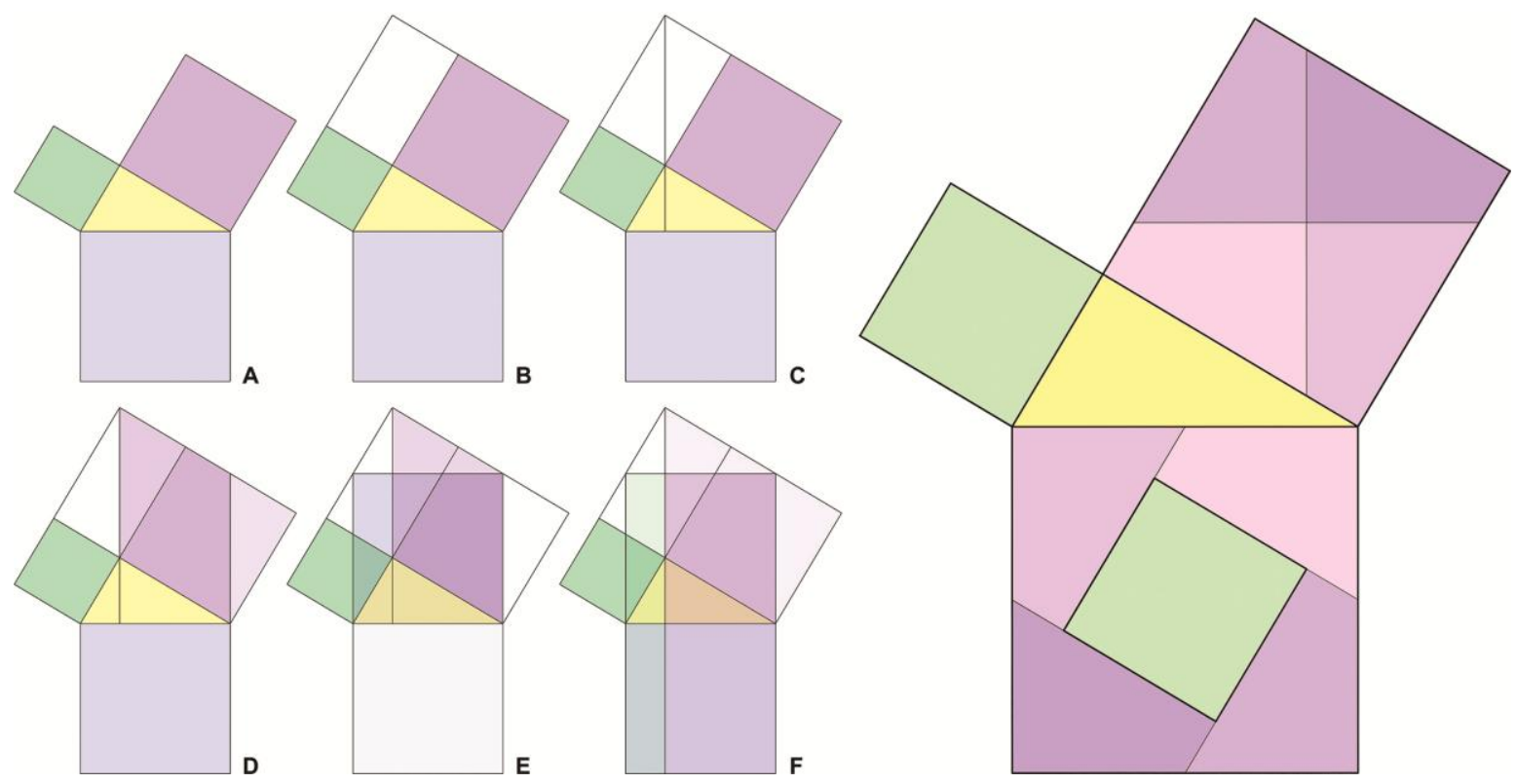

Figure 1. (A-F). Pitagora's teorem, dimostration of Euclide. (G) Pitagora's teorem, dimostration of Perigal.

The position of the two squares above suggests that their sides be extended (Figure 1B). This initial step recommends the construction of the vertical line that passes through the common vertex of the two squares and is perpendicular to the hypotenuse of the yellow right triangle (Figure 1C). It is easy to see that the triangle produced by extending the right side of the blue square is identical to the triangle to the left of the upper purple square. Therefore, the rhombus created by moving the triangle added to the remaining portion of purple square, has the same surface area as the original purple square (Figure 1D).

Recognizing a similarity in the drawing between the extension of the blue square and its side, it is possible to slide the blue square up. Once again the drawing suggests an equivalence between the upper triangular portion of the rhombus and the portion of the lower yellow triangle (Figure 1E). We can therefore slide the triangular part of the upper rhombus down until it rests on the lower side of the blue square, thereby obtaining a rectangle. It is thus demonstrated that the portion of blue 
square delimited by the first line constructed, has a surface area equal to that of the purple square built on the right side of the right triangle (Figure 1F). It is also possible to make an analogous construction for the green square, thereby demonstrating that the blue square built on the hypotenuse has an area equal to the sum of the areas of the purple and green squares built on the sides. It is then the task of the mathematician to demonstrate the validity of the suggestions and intuitions using the appropriate mathematical tools.

It is not hard to see how the drawing itself suggests possible alternative solutions to the problem. Each graphical sign becomes a tool that stimulates intuition, imagination, and the emotion of a probable positive solution. It is a communicational system wherein the drawing actively participates in the definition and resolution.

Another example of the Pythagorean theorem can be taken from the demonstration published in 1874 by Henry Perigal (Figure 1G). Perigal's intuition was to move the green square built on the smaller side of the triangle to the centre of the larger square built on the hypotenuse. This operation suggests that by appropriately dividing the area remaining after subtraction of the green square and recomposing the parts, the square built on the second side is formed. Extending the sides of the internal square in one direction, the area is divided symmetrically. The extension of the sides can occur either to the right or left; in the latter case there is no solution to the problem, but in the former, recomposing the areas yields the square built on the longer side. It seems rather unlikely that this operation occurs entirely in the mind of Perigal, who, only through imaginative effort, was able to presume the geometrical conditions without the aid of a drawing. Again the image suggests a possible solution that the open mind identifies and uses to obtain the solution to the problem. Intuition is anyway determined by emotional conditions and an imaginative predisposition. It is unlikely that a person not versed in mathematics will find the solution to a mathematical problem. Therefore, it is unlikely that one can solve a geometrical problem without the use of a drawing.

Another example of a different nature no longer deals with geometry, but with colour. Only in 1961 did Johannes Itten publish The Art of Color, the result of a life of research that, drawing on the work of Goethe, Runge, Berzold, and Chevreul, proposes a practical theoretical approach to the use of colour. Itten's theories have radically changed the traditional and unsophisticated relationship with colour, demonstrating how one can have a rational, objective relationship with it, thereby modifying contemporary culture, from design to cinema, from publicity to fashion.

One of the first tables found in The Art of Color is the twelve-part chromatic disk (Figure 2). With this tool, Itten demonstrates how it is possible to identify the relationships between complementary colours and the harmonic agreement of colours through the geometries of the equilateral and isosceles triangles, the square, and the rectangle.
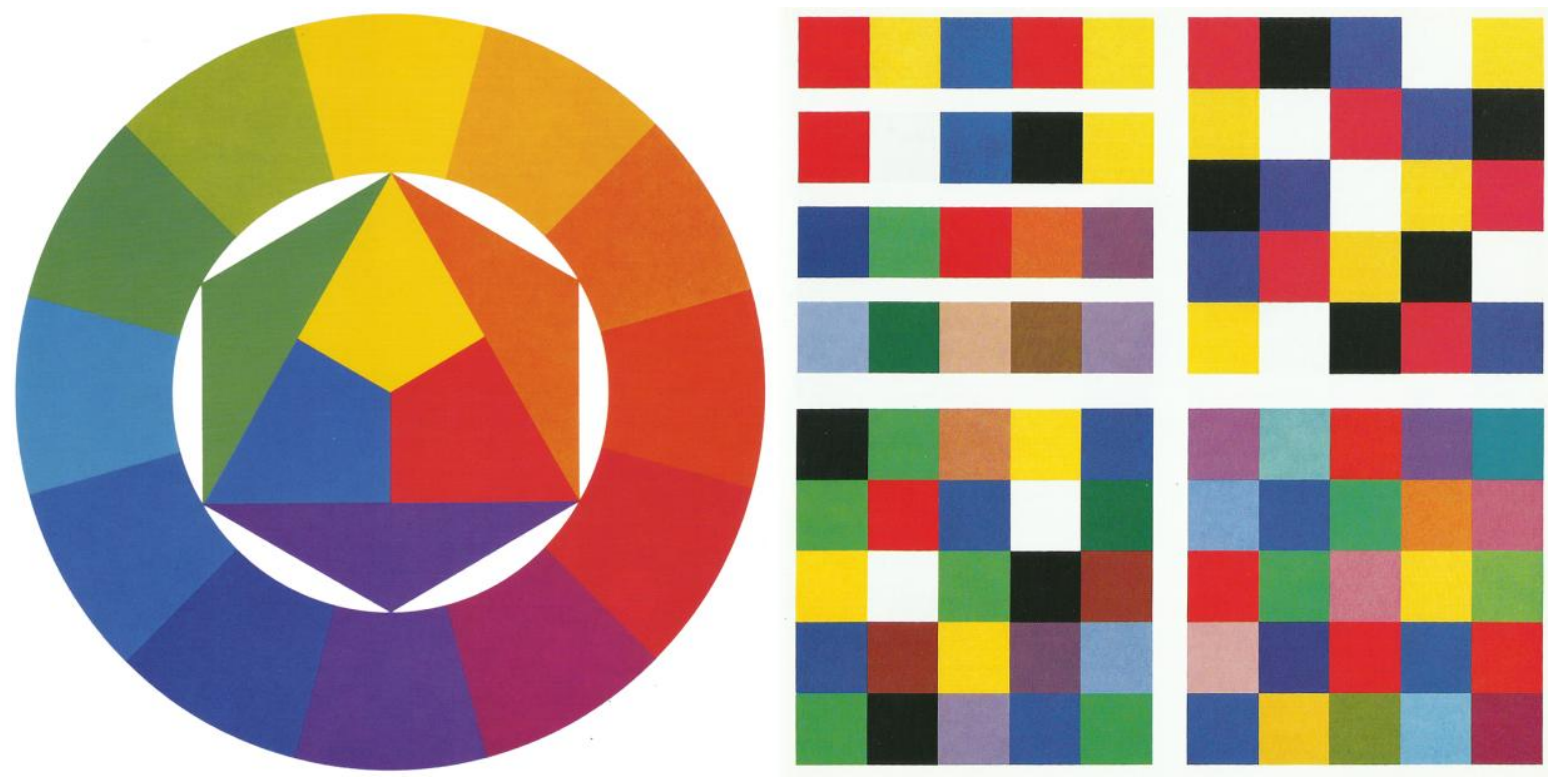

Figure 2. Johannes Itten. The chromatic circle, some exercises on the contrast of pure colors. 
Those who have tried to construct Itten's chromatic circle have seen that this theoretical approach, which is widely accepted, runs into practical problems when mixing colours. Even when using the primary colours defined by a single production house, one discovers that while they generate the medium grey expected by the theory when mixed together, the composition of the secondary colours does not always give the expected results. If a given yellow and blue are right for creating green, the same yellow is not right for making orange and the blue is not right for making purple. Some of the secondary colours are dirty, that is, they tend towards grey.

The chromatic circle discretizes a continuum, a flow between the colours. Itten sews together the ends of the visible spectrum, which ranges from 400 to $700 \mathrm{~nm}$, and uses the twelve portions to schematize and synthesize the continuous flow between the colours. This means, however, that the primary colour blue is the point to which purple and green tend. It is, therefore, nondimensional. We are thus forced to confirm that the primary colours do not exist; they are just points to which the secondary colours tend.

This consideration undermines Itten's theory of colours, disputing the evident principle of the existence of primary colours on which all the other colours depend. At any rate, one cannot deny that the importance of the theory lies not in its assumptions but in the harmonic and complementary relationships between the colours determined therein. It is therefore necessary to integrate the formulation with limiting points to which the secondary colours tend, effectively changing the scheme proposed by Itten but maintaining the successive considerations as valid.

The drawing should help to solve the problem by proposing a new structure determined by three parabolas that tend towards the limiting points of the primary colours (Figure 3). This structure still allows Itten's considerations regarding complementarity to be realized, along with the harmonic agreements of the colours again determined by equilateral and isosceles triangles, squares, and rectangles.

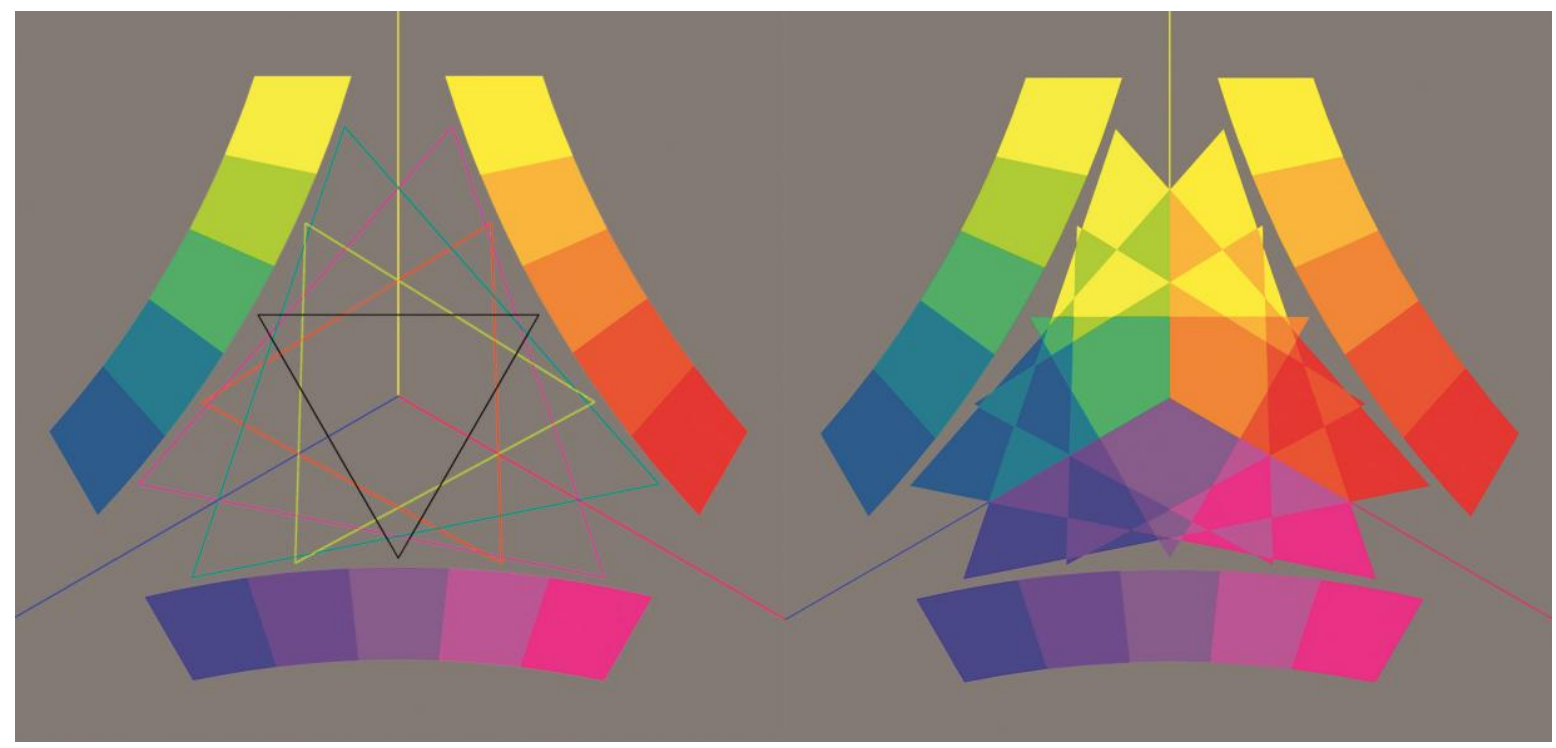

Figure 3. Parabolic distribution of colors.

The best expression of the relationship between image and creator can be found in project drawings. From the sketch to the technical drawing to the drawing made to convince clients of the intentions, the image demonstrates all the possibilities that can be made at the hands of the designer. An impressive number of images are made, of which, unfortunately, only a very few will be archived; the vast majority will be discarded because they will have been surpassed and are no longer useful to the descriptive goals of the now finished work. All of this material would be exceedingly useful for understanding the design process and the communicational relationship between the designer's thought and the image.

Looking through a monograph about Le Corbusier for an example that shows this relationship between designer and drawing, I found with some difficulty some drawings of the Heidi Weber 
Museum, which would later become the Pavillon Le Corbusier in Zurich [7] (pp. 142-157). These images give a partial hint of the dynamic relationship between the drawing and designer.

The idea of the design is to formally and physically distinguish the two functions of the building: the volumes dedicated for exposition and organized in various ways are different from the functional surfaces of the roof. The building results from a composition of cubes, each of which is covered by an independent roof: two square umbrellas connected along one edge. Each of these, by dividing the square along an axis, gives rise to the four inclined parts of the roof supported by pillars situated on the axes (Figure 4).
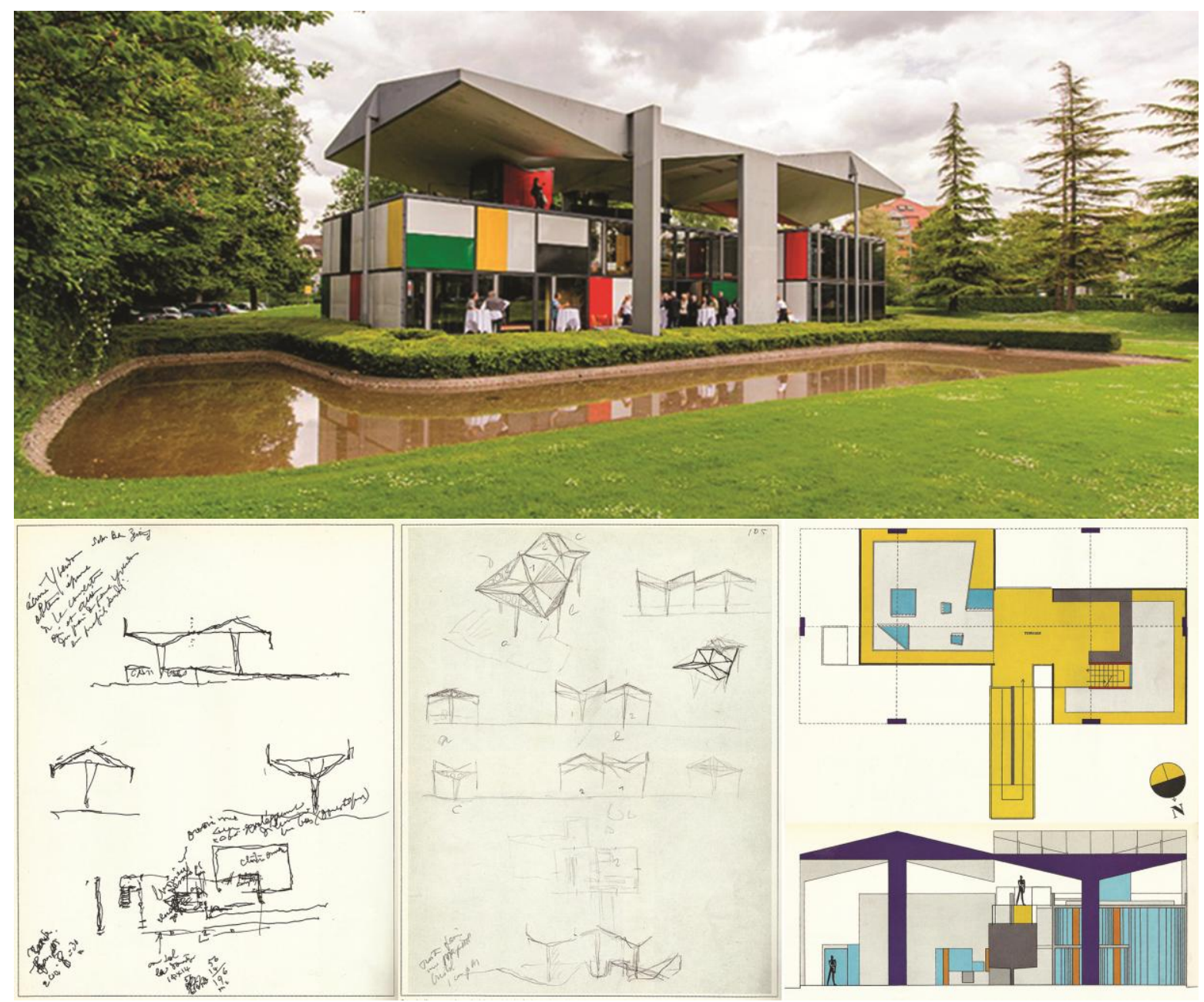

Figure 4. Pavilion Heidi Weber, Center Le Corbusier, Zurich. First sketches, studies on the geometries of the two covers and supporting pillars. The relationship between the roof structure and the architectural spaces - plant and section.

The first sketch already demonstrates the compositional idea of the roof. The two structures are connected and supported by triangular pillars placed on a horizontal base. Below, two sketches depict the two separate structures. Further below, a first draft of the plan hints at the horizontal base with the projection of the roof and the position of the pillars. In these drawings, it is the layout of the internal panelling that delimits the exposition space.

In the subsequent axonometric drawing, Le Corbusier develops the desired spatial condition to compose the planes of the roof. He clarifies the relationship between the two umbrella structures through additional schematic perspectives, but their spatial meaning is already clear. Below, connected to it in projection, the scheme of the plan is still seen. The complete spatial condition of the object is demonstrated through these rough drawings.

The two subsequent tables were chosen from among the seven that describe the project presented in the monograph (Figure 5). The large roof structure is identified by the two $\mathrm{T}$ united with 
the colour blue which cover the parallelepiped volume of the expository space, demonstrating the formal distinction between the two uses. To the right of the axis, the dark grey colour is the stairway connecting the different floors. It is likely that this juxtaposition of the stairway, which is formally independent because it is inclined and lies beyond the roof, leads Le Corbusier to reflect and make a substantial modification. In the drawing we see that the architect decides to separate the two roof structures with a plane corresponding to the stairs, which becomes the formal axis of the entire composition.
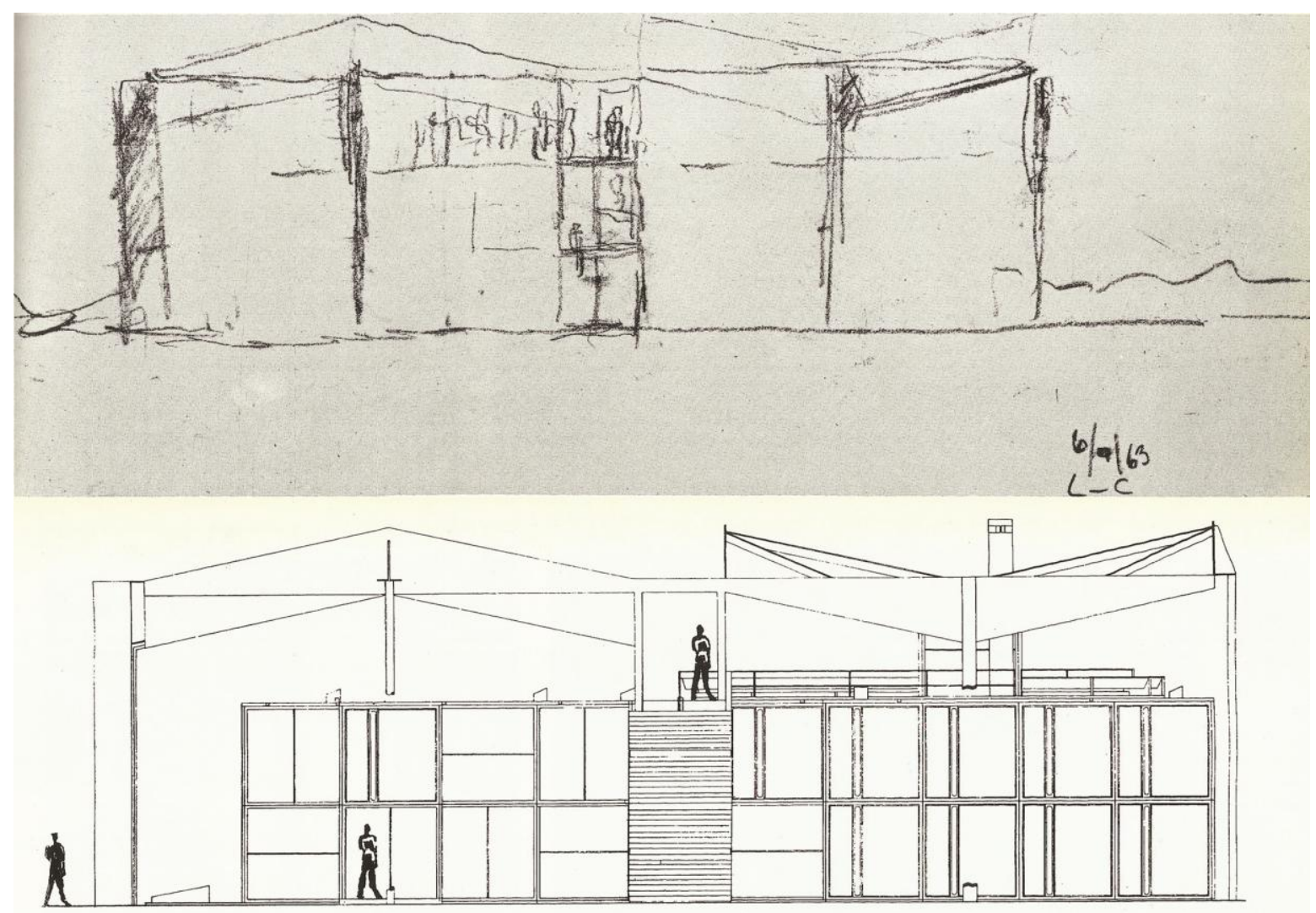

Figure 5. Sketch and definitive solution with new roof positioning compared to the ramp.

This rethinking, suggestion, reflection, consideration is the fruit of the relationship that the image has with the mind of the designer. A doubt is present, but the same drawing immediately indicates the terms for its resolution. Intuition is checked and modified until a new conversational structure is built that is inevitably reflected in the user, who will experience those spaces and become emotionally tied to the author through the work.

Vasari tells us that Leonardo worked on the portrait of the Mona Lisa for four years; Giacomo Leopardi worked on his poem "L'Infinito" for three years.

This is perhaps an outrageous, excessive juxtaposition; the two works are separated by about three hundred years. In both, however, we discover an entirely particular condition, an emotional relationship that connects us completely and uniquely with the physical concreteness of their being. We experience a precarious balance in a borderland between what is seen and not seen, what is written and not written, between the reality of those brush strokes on a canvas and the words written on a sheet of paper and the imagination and emotions that they evoke. Both works were made concretely and are presented as postulates in a geometrically demonstrated theorem. Leopardi responds to the objective concreteness of the portrait by beginning his poem with the word "always", both absolute and clear truths. And we experience that entirely universal and personal condition of "comparing that infinite silence to this voice" and we still singularly experience our "thought drowning in this immensity: and sinking would be sweet in such a sea". 
It is a tie that inextricably links the author to the user through the work. And the work, when it is art, creates the indissoluble connection between signifier and sign, surpassing both and giving us the possibility of sensing other infinities, imagining them. We become emotionally involved in a relationship that, while confirming what we have previously experienced, is ever new, activated by our continual changes and moods. At any rate, it is one thing to look at a drawing; it is entirely another to make a drawing.

\section{Conclusions}

Continuing with this equivalence between language and image, a question arises: what would happen if an individual stopped using language? That is, what would happen if at a certain age he stopped using words not only to communicate to others, but stopped talking to himself, i.e., if he limited himself simply to listening, no longer trying to express and think with words? Slowly but inexorably his imaginative and judgement capacities would be lacking, he would break that active relationship with thought, and would probably still be able to relate to the world, but it would be completely unidirectional; he would react to stimuli without a real capacity for judgement. He would find himself living according to instinct and learning would be determined by strategies for survival.

This is partly what happens with the drawing.

At a certain point in their growth, babies discover the relationship between gestures and the signs left on a surface. At around two years of age they are able to control the signs made on a sheet of paper. They connect the name pronounced for a drawing to the object indicated by the adult. They build the first relationships between graphical representation and the concept.

At a certain point, even if the graphical characteristics and signs on the sheet of paper are absolutely like those made the preceding day, the child gives a name to her drawing. Whether this is due to the recognition of similarities with the mental model or to please the adult, the role of the drawing has changed fundamentally; the child realizes that her drawing can resemble something. The child changes her design, integrates it with voluntary signs, corrects and modifies. A circle becomes a head; other segments become the arms and legs.

At around five years of age, the depicted person is completely formed. The head has a mouth and nose and is separated from the torso by the neck; the arms have hands and the legs have feet. Colours are used to distinguish the individual parts or one person from another. The entire compositional space is used, the signs are distributed freely on the paper, but the people remain mostly vertical. Other elements begin to appear: the Sun, a house, a tree.

Drawings are associated with the linguistic system, increasing the conceptual symbolic aspect: boys have trousers and girls have long hair and skirts; the house has a triangular roof and the tree has green foliage. The compositional elements are distributed in a two-dimensional space or situated on the ground or high in the sky. The child becomes increasingly able to draw, leading to a greater formal overall synthesis corresponding to the enrichment of particular details: the house has open windows with curtains and there are flowers in the grass. Children draw what they know, not what they see, and therefore the walls of the house are transparent to see what is inside; the tree is drawn with its roots.

Up to eight or nine years of age, drawings are a way to tell stories. It is an imaginary world, a seductive game of continuous invention. Later, probably to please adults, children begin to imitate adults' drawings, or better, the drawings made by adults for children (i.e., Peppa Pig), thereby breaking that independent and creative communicational relationship with the drawing.

For the first few years of life up to 10-12 years, children build, imagine, narrate, check, and develop their own thought with drawings, but they abandon them unexpectedly, settling for just the verbal linguistic system, probably because it is more common and has a greater chance of success. This is also because it is used by adults, who in turn have abandoned drawing. Recalling Wallon, Daniel Widlöcher states, "children copy little from reality, while adolescents in contrast no longer express a fantasy life in drawings. The appearance of visual realism is simultaneous with the decline of the drawing" [8] (p. 44). 
Right when children begin to appreciate the difference between written and spoken language, when they master the distinction between formulating thoughts by speaking to themselves and socializing the thought with words, when they begin to appreciate the distinction between similar words, children stop drawing and the drawing becomes only the listless response to an educational request.

Only a few will continue to use drawing through a fundamentally autonomous and solitary relationship. Even fewer will follow artistic studies, encountering a distorted idea of academic drawing that has not comprehended the teaching of the masters, drawing the student into the conviction that the scope of drawing is to draw from reality, that is, make a copy of it. The situation recalled by Loquet is thus confirmed, that for drawing, only what is seen can overlook any intellectual consideration, thereby negating the communicational role between the image and thought.

\section{References}

1. Bergson, H. Materia e Memoria; Laterza: Rome, Italy, 1996; ISBN 88-4204894-1.

2. Zeki, S. Splendori e Miserie del Cervello; Codice: Turin, Italy, 2010; ISBN 978-88-7578-165-1.

3. Biederman, I. Recognition-By-Components: A Theory of Human Image Understanding. Psychol. Rev. 1987, 94, 115-147

4. Langer Susanne, K. Abstraction in art. J. Aesthet. Art Crit. 1964, 22, 379-392

5. Goodman, N. I Linguaggi dell'arte; Il Saggiatore: Milan, Italy, 2013; ISBN 978-885650347-0.

6. Felice, C. Against Mental Images. Language and Thought between Nature and Culture. Collegio San Carlo di Modena 19022016 Available online: http://www.fondazionesancarlo.it/conferenza/contro-le-immaginidella-mente/ (accessed on 11 November 2017).

7. Willy, B. Le Corbusier; Les Editions d'Architecture Artemis Zurich; Birkhäuser Verlag: Basel, Switzerland, 1970.

8. Widlocher, D. L'interpretazione dei Disegni Infantili; Armando Editore: Rome, Italy, 1965; ISBN 8871443160.

(C) 2017 by the author. Licensee MDPI, Basel, Switzerland. This article is an open access article distributed under the terms and conditions of the Creative Commons Attribution (CC BY) license (http://creativecommons.org/licenses/by/4.0/). 